\title{
PAPER
}

\section{The effect of a mixing console on the monitoring response in a mixing room}

\author{
Masataka Nakahara $^{1, *}$, Akira Omoto ${ }^{2, \dagger}$ and Kyoji Fujiwara ${ }^{2,+}$ \\ ${ }^{1}$ SONA Corporation, 2-19-9 Yayoi-cho, Nakano-ku, Tokyo, 164-0013 Japan/ \\ Kyushu Institute of Design, Shiobaru 4-9-1, Minami-ku, Fukuoka, 815-8540 Japan \\ ${ }^{2}$ Faculty of Design, Kyushu University, \\ Shiobaru 4-9-1, Minami-ku, Fukuoka, 815-8540 Japan
}

(Received 28 June 2004, Accepted for publication 27 October 2004)

\begin{abstract}
Mixing consoles, which are usually of large dimensions, are essential equipment in a mixing room of a production studio. This large obstacle always has to be placed nearby the listening position in a mixing room. A significant dip at around $100 \mathrm{~Hz}$ is often observed on the monitoring response, the transfer function from the loudspeaker to the listening position, in a small/medium sized mixing room. This paper studies the relationship between the dip at around $100 \mathrm{~Hz}$ and the effect of the presence of the mixing console. The generating mechanism of the dip is investigated by numerical simulations and experimental measurements. The results show that the reflection sound from the floor and the behavior of the mixing console as a cross over filter are important when considering the dip. Examples of trials for improvement of the dip are also examined.
\end{abstract}

Keywords: Studio, Mixing room, Console, Response, Dip

PACS number: 43.55.Br, 43.20.El [DOI: 10.1250/ast.26.90]

\section{INTRODUCTION}

In the acoustic design of a mixing room, it is important that the monitoring response, namely the transfer function from the loudspeaker to the listening position, has flat amplitude characteristics on the frequency domain. In reality, the frequency response need not be entirely flat, however strong dips and peaks should be avoided. However, significant dips at around $100 \mathrm{~Hz}$ are often observed in mixing rooms [1,2]. Figure 1 shows the examples of the monitoring responses in four different types of the mixing rooms [2].

We find the strong dips at around $100 \mathrm{~Hz}$ in the responses shown in Fig. 1, and same kind of the dip is also shown in another reports e.g., [3]. This kind of dip often has harmful effects on the mixing work, and is usually explained as being the results of the following physical phenomena.

(1) Isolated room modes and their interferences.

(2) Interference between the direct sound and a strong early reflection sound.

These ideas have been incorporated as one of the basic techniques for the acoustic design of a mixing room e.g.,

\footnotetext{
*e-mail: nakahara@sona.co.jp

†e-mail: omoto@design.kyushu-u.ac.jp

+e-mail: fujiwara@design.kyushu-u.ac.jp
}

[4]. However, from experience, the author postulates that the dips cannot be explained by such conventional theories. Focusing on the monitoring (listening) environment in a mixing room, the mixing console always has to be placed nearby the listening position (Fig. 2). The dimensions of the mixing console are too large for it to be regarded as the acoustically transparent for a $100 \mathrm{~Hz}$ sound wave. In this study, the author focuses on this point, and examines the relationship between the dip at around $100 \mathrm{~Hz}$ and the effect of the presence of the mixing console. The final goal is to clarify the generating mechanism of the dip and to remove the dip from the monitoring response.

\section{ANALYSIS MODEL}

\subsection{Examined Field}

The field examined is assumed to be a two-dimensional $(y-z)$ sound field based on the vertical section of a monitoring environment in a mixing room. Figure 3 shows the modeled sound field both for numerical analyses and scaled model measurements. Because of the two-dimensional model, cross section and surface conditions are uniform along the $x$-axis. Therefore, mapped to the threedimensional environment, the source is regarded as an infinite coherent line source along the $x$-axis.

In this field, room boundaries are omitted excluding the floor, because walls and ceilings of a mixing room are 


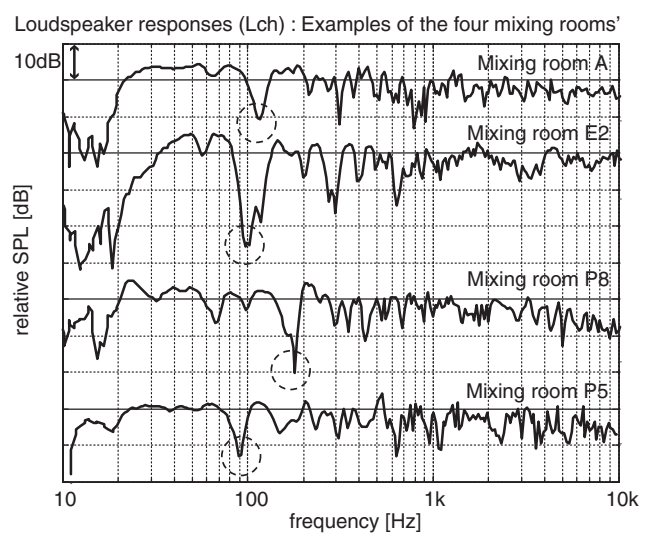

Fig. 1 Examples of the monitoring responses in the four mixing rooms.

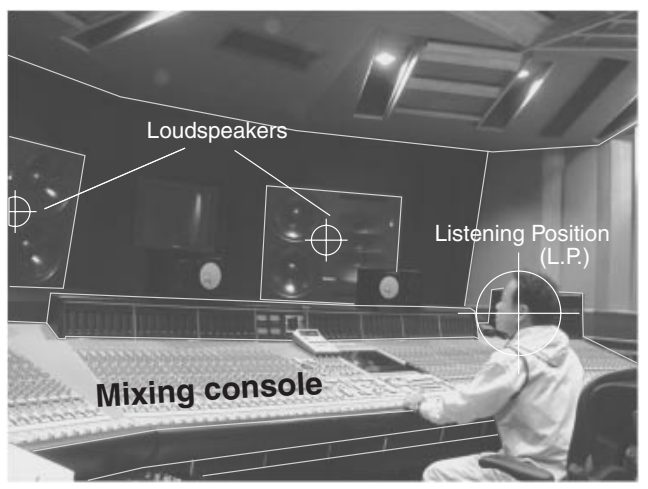

Fig. 2 A typical monitoring environment in a mixing room. Loudspeakers (sources), the mixing console and the listening position (L.P., receiver).

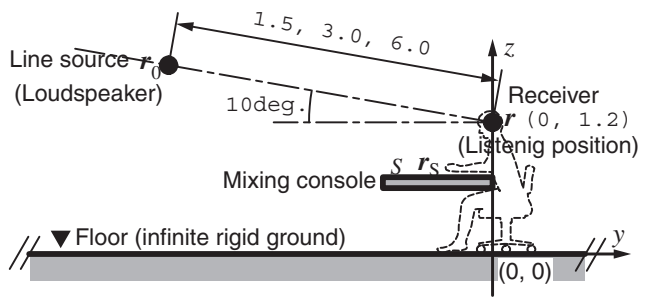

Fig. 3 The examined field. Two-dimensional sound field based on the vertical section of a monitoring environment in a mixing room.

usually finished with materials that absorb enough sound [5]. A field without room boundaries is also good for understanding the basic behavior of a mixing console.

The listening position (L.P., the receiver) is located at the edge of the mixing console, $(y, z)=(0,1.2)$, where is usually defined as the reference position in a mixing room.

The monitoring distance, namely the distance from the receiver (listening position) to the source (loudspeaker), is usually around $3 \mathrm{~m}$ in a small/medium sized mixing room $[6,7]$. In respect to the height of the source, the loudspeaker is usually placed at higher position than the listening

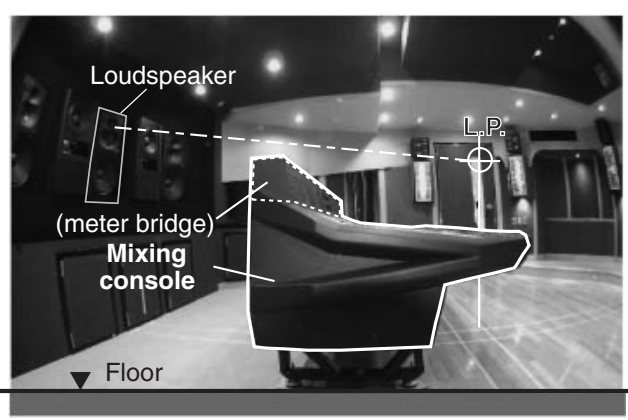

Fig. 4 Side view of the mixing room; Loudspeaker, Mixing console (meter bridge), Listening position.

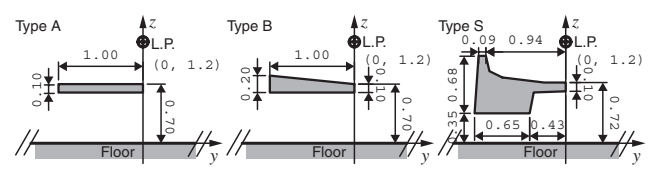

Fig. 5 Three types of a mixing console are examined.

position to avoid shadowing the direct sound by the meter bridge of a mixing console whose top height is around $1.1 \mathrm{~m}$ from the floor. (Fig. 4). As the result, in most mixing rooms, the loudspeaker is placed at the height where the elevation angle is around 10 degrees from the listening position [7]. Therefore, in the examined field, the source is located at the position where the distance from the L.P. is 3 meter and the elevation angle is 10 degrees. Other two distances, 1.5 meter and 6 meter, are also assumed to examine the effect of the source positions (Fig. 3).

In terms of the usability of the mixing works, follows are common dimensions of the sectional shape of the mixing consoles.

- The height of the top surface is approximately $0.7 \mathrm{~m}$ from the floor.

- The depth is shorter than $1.5 \mathrm{~m}$, and is usually approximately $1 \mathrm{~m}$.

On the other hand, follows are unique dimensions for various types of the mixing consoles.

- The shape of the top surface. e.g., straight, angled and more complex form.

- The thickness, in other words, air space between the floor and the bottom of the mixing console.

- The height of the meter bridge and its depth.

Taking account of the matters above, three kinds of the mixing console's shape are examined (Fig. 5).

- Type A; the simplified model for analysis of the basic behavior of the mixing console.

- Type B; angled top surface.

- Type S; the shape based on the real mixing console shown in Fig. 4. Type $S$ has a meter bridge, a complex shaped top surface and a thick body. More than 100 real 'Type $S$ ' mixing consoles and more than 3,000 
same kind of the mixing console are working in the world.

\subsection{Numerical Analysis}

Following examinations are conducted in this study.

- The changes of the monitoring responses due to the different shapes of the mixing consoles and by the source positions.

- The variation of the sound field caused by the presence of the mixing console.

- Contributions from the real source and from the imaginary source.

- The effect of the absorption of acoustical boundaries on the monitoring response.

In order to examine them with enough accuracy, the author studies them based on the results of the numerical analysis via the boundary element method (BEM). For the calculation, the maximum length of the element boundaries was set to be $\lambda / 8$ ( $\lambda$; acoustic wave length). When the mixing console is removed, namely the hemi-free sound field condition, numerical calculations are made by the summation of the Hankel functions without the BEM procedure.

\subsection{Measurement}

To confirm the validity of the numerical calculation via the BEM, 1/10 scaled measurement was conducted in a two-dimensional anechoic chamber (Fig. 6). The chamber has a $2.65 \mathrm{~m} \times 1.67 \mathrm{~m}$ sound field. A two-dimensional free sound field can be obtained up to $6.3 \mathrm{kHz}$ in the chamber due to the thickness, $0.025 \mathrm{~m}$, of the chamber. A tweeter

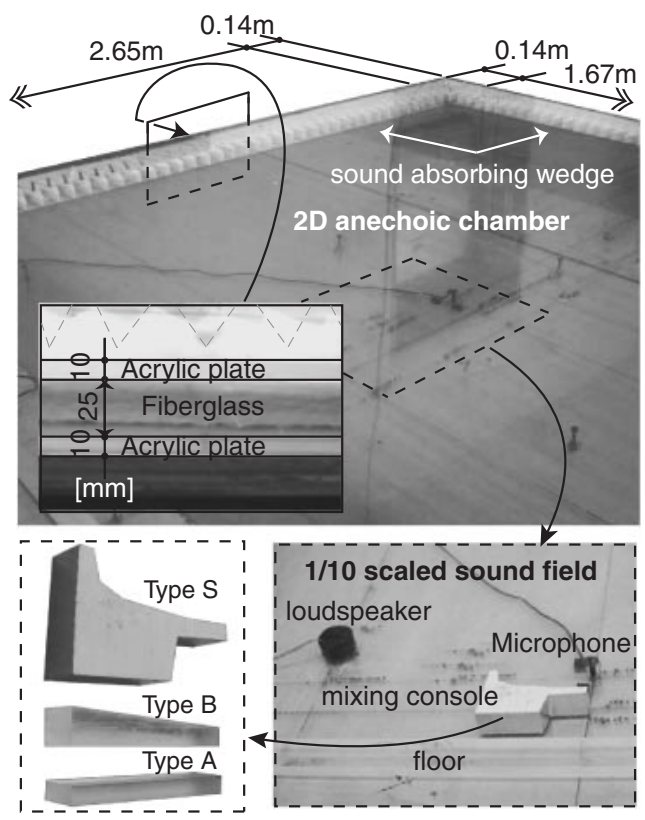

Fig. 6 The 1/10 scaled measurements in the twodimensional anechoic chamber.

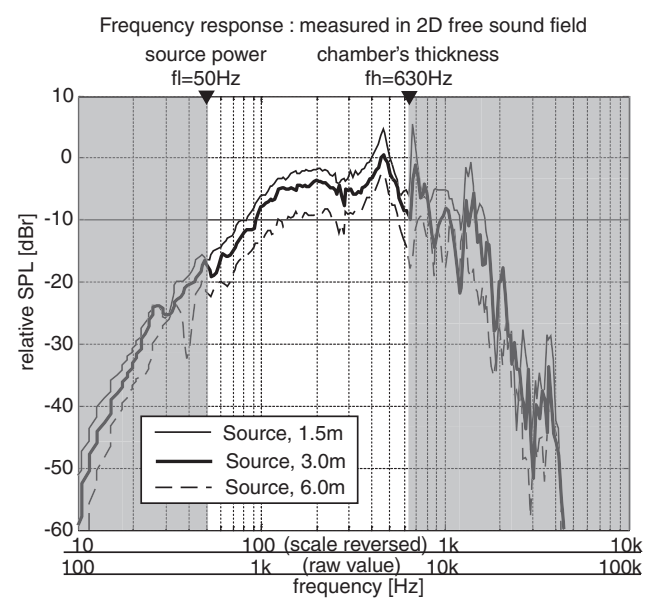

Fig. 7 The source responses in the two- dimensional anechoic chamber.

driver was put in the chamber as a source. Figure 7 shows the source responses at $1.5 \mathrm{~m}, 3.0 \mathrm{~m}$ and $6.0 \mathrm{~m}$ distances from the receiver. In terms of source characteristics and chamber performance, the range from $50 \mathrm{~Hz}$ to $630 \mathrm{~Hz}$ is the confidence frequency as the scale reversed frequency. The floor and the 1/10 scaled mixing consoles, Type A and Type B, were made from solid acrylic. Type $S$ was made from plywood with thin aluminum sheeting on the cut ends. The tweeter driver, the microphone, the mixing consoles and the acrylic floor were arranged on the 1/10 scaled field in the chamber, and impulse responses were measured using a TSP (time stretched pulse) signal with a $96 \mathrm{kHz}$ sampling frequency.

\section{RESULTS}

\subsection{Effect of the Shape of a Mixing Console}

The effects of the different shapes of the mixing consoles were examined via the BEM. Figure 8 shows the examined sound field. The source is fixed at $3 \mathrm{~m}$ distance from the receiver, and three different types of the mixing consoles, Type A, Type B and Type S, are examined. The boundary admittances of the mixing consoles were assumed to be zero for numerical calculations. The response in a hemi-free sound field, which is nothing but a floor, was also examined.

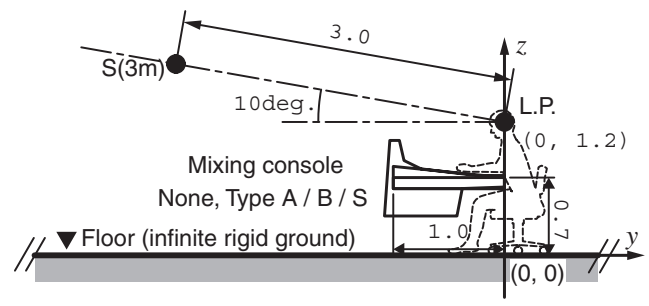

Fig. 8 The modeled field for the results shown in Figs. 9 and 10 . 


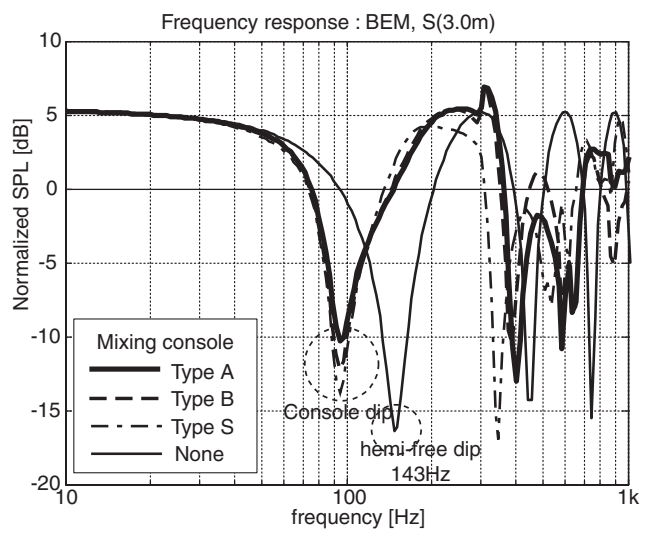

Fig. 9 Frequency responses at the L.P. with the three types of mixing console. Responses were calculated at $1 / 24$ octave intervals via BEM. The $0 \mathrm{~dB}$ indicates the free sound field response.

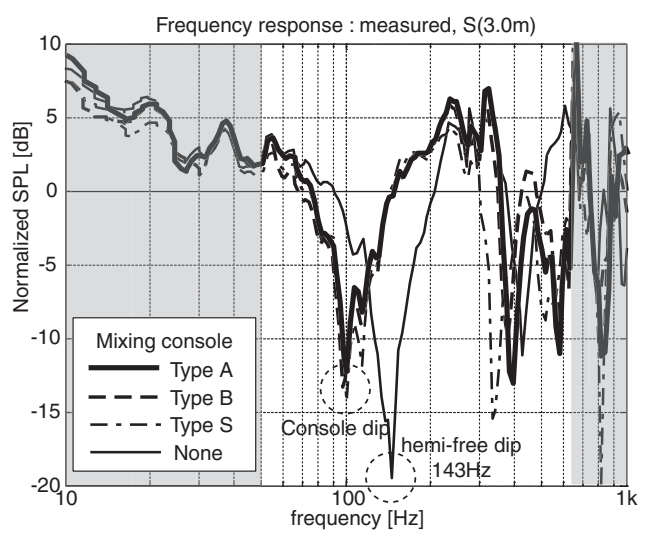

Fig. 10 Frequency responses at the L.P. with the three types of mixing console. Responses were measured in the 2D anechoic chamber. Averaged data are plotted at $1 / 24$ octave intervals. The $0 \mathrm{~dB}$ indicates the free sound field response. The gray area indicates areas of no confidence.

Figure 9 shows the results via the BEM, namely the difference of the response due to the shapes of the mixing console. To confirm the validity of the numerical calculation, the results via the scaled measurement are also shown in Fig. 10. Results are normalized by the free sound field response. Calculation was conducted at $1 / 24$ octave frequency intervals. Measured results are plotted as the averaged value at $1 / 24$ octave frequency intervals. The gray area in Fig. 10 indicates areas of no confidence.

Comparing the simulated (Fig. 9) with the measurement (Fig. 10) results, both results correspond enough well to confirm the validity of the calculation method. In respect to differences of the consoles' shapes, purposive differences are not found at a low frequency. All three types give the same kind of the strong dip to the frequency response at approximately $100 \mathrm{~Hz}$. Therefore the examinations of Type
B and Type S are omitted from the next section.

In the response without the mixing console, a strong dip appears at $143 \mathrm{~Hz}$ caused by the path difference between the direct sound $(3.0 \mathrm{~m})$ and the reflection sound from the floor $(4.2 \mathrm{~m})$. This strong dip at $143 \mathrm{~Hz}$ seems to move to a lower frequency, approximately $100 \mathrm{~Hz}$, when the mixing console is inserted. This result suggests that some kind of variation occurs in the sound field when the mixing console is inserted.

\subsection{Difference of the Source Position}

The effects of the source positions were also examined via the BEM. Figure 11 shows the examined sound field. The Type A mixing console was positioned, and three different positions of the source, $1.5 \mathrm{~m}, 3.0 \mathrm{~m}$ and $6.0 \mathrm{~m}$ from the receiver, were examined.

Figures 12 and 13 show the results via the BEM. To confirm the validity of the numerical calculation, the $1 / 10$ scaled measurements are carried out and the results are shown in Figs. 14 and 15. Figures 12 and 14 show responses when the mixing console is present, and Figs. 13 and 15 show the responses when it is not. Conditions for the calculations and the measurements are same as those in Sect. 3.1.

Comparing the simulated (Figs. 12 and 13) with the measurement (Figs. 14 and 15) results; they correspond

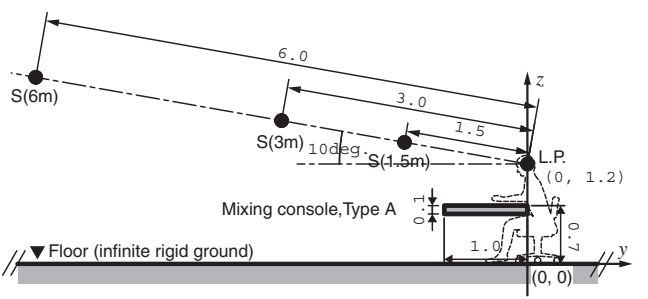

Fig. 11 The modeled field for the results shown in Figs. 12 to 15 .

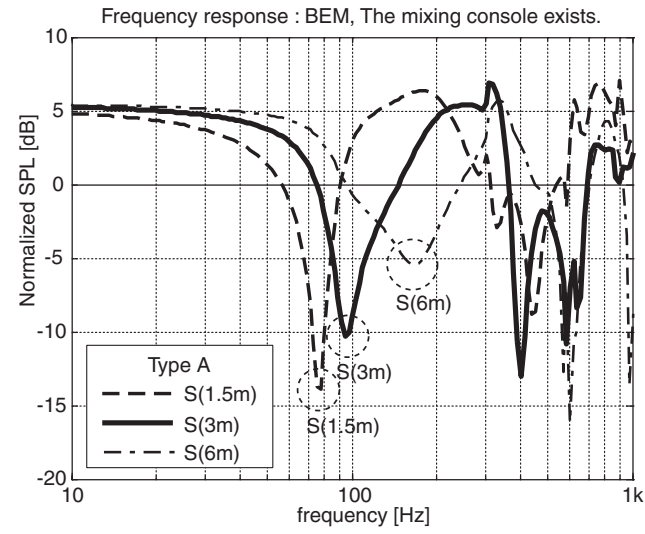

Fig. 12 Calculated responses from three different source distances for the Type A mixing console. 


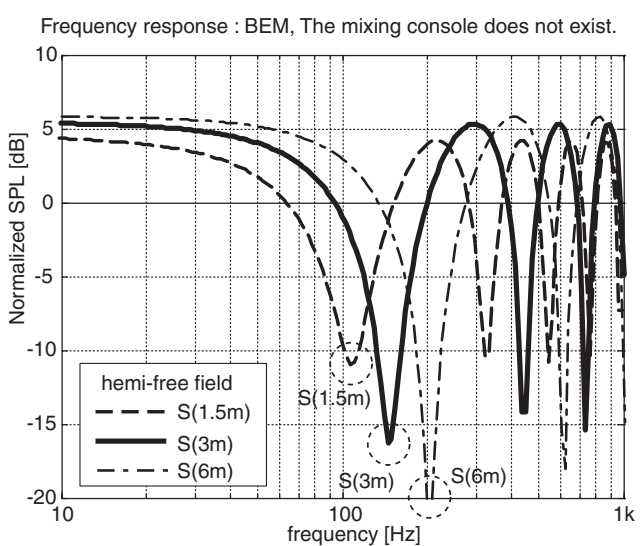

Fig. 13 Calculated responses from three different source distances with no mixing console.

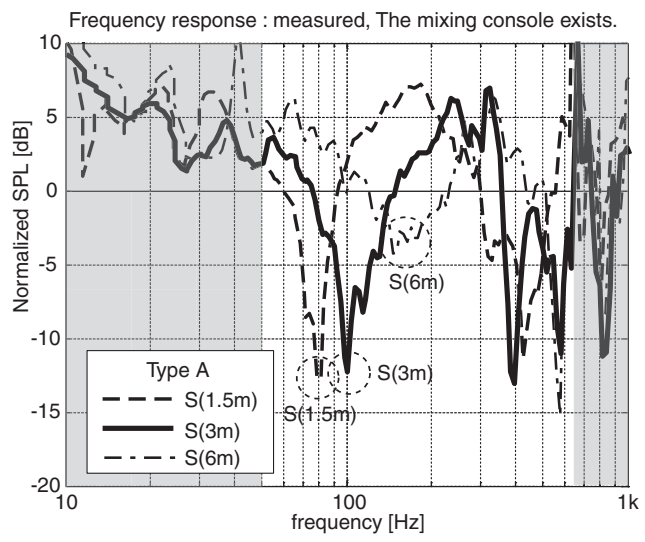

Fig. 14 Measured responses from three different source distances for the Type A mixing console.

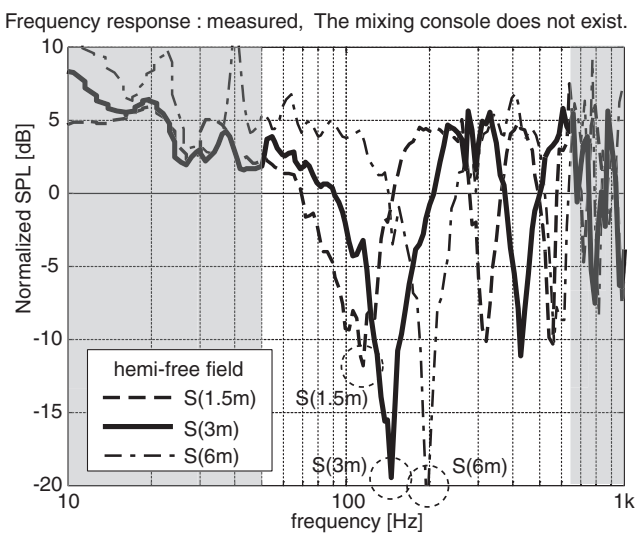

Fig. 15 Measured responses from three different source distances with no mixing console.

well enough to confirm the validity of the simulation method. According to the results without the mixing console (Fig. 13), the farther out the source is located, the higher the lowest frequency of the dip moves because of the variation of the path difference between the direct sound and the reflection sound from the floor. This tendency is also observed in the results with the mixing console (Fig. 12). This suggests that interference between the direct sound and the reflection sound from the floor contributes to the dip in the low frequency regardless of the presence of the mixing console.

However details of the appearances of the dips are different in the two results. First, the dip's frequencies with the mixing console (Fig. 12) do not correspond with those without the mixing console (Fig. 13). Second, quality factors ( $\mathrm{Q}$ factors) show different characteristics. When the mixing console is not present (Fig. 13), Q factors of the dips are almost consistent regardless of the source position. On the other hand, when the mixing console is present (Fig. 12), the farther out the source is located, the smaller the $\mathrm{Q}$ factor of the dip becomes. Therefore, the mixing console clearly initiates some kind of acoustical effect on low frequency responses. And the results also suggest that the dip affected by the mixing console necessarily occurs at around $100 \mathrm{~Hz}$ in the small/medium sized mixing rooms, even though all walls and the ceiling are absorbed perfectly.

\section{DISCUSSION}

\subsection{Variation of the Sound Field with the Mixing Console}

To examine the effect of the mixing console on the sound filed, spatial distributions of the sound pressure levels were calculated. Calculation was conducted for $50 \mathrm{~Hz}, 75 \mathrm{~Hz}, 100 \mathrm{~Hz}, 150 \mathrm{~Hz}$ and $200 \mathrm{~Hz}$ at $0.1 \mathrm{~m} \times 0.1 \mathrm{~m}$ mesh points. The boundary admittance of the mixing console is assumed to be zero, and the source is located at $3 \mathrm{~m}$ distance from the receiver. Figures 16 and 17 show results without or with the mixing console, respectively.

Figure 16 presents the interference pattern created by the real source and an imaginary source against a floor; features are:

(1) No nodal line of the sound pressure level (SPL) is found at $50 \mathrm{~Hz}$, but appears at $75 \mathrm{~Hz}$

(2) As the frequency become higher, the nodal line becomes thin and comes down to the floor.

(3) The L.P. is involved with the nodal line at approximately $150 \mathrm{~Hz}$. The dip in the frequency response without the mixing console (Fig. 13, S(3 m)) reflects this state.

Comparing Fig. 17 with Fig. 16, some features of the sound field with the mixing console are the same as without it. However, with the mixing console, some differences can be observed in the SPL distribution:

(1) Part of the nodal line almost adheres to the surface of the mixing console at $75 \mathrm{~Hz}$.

(2) At a frequency higher than $100 \mathrm{~Hz}$, the nodal line is split into two segments, and the edge of the one 


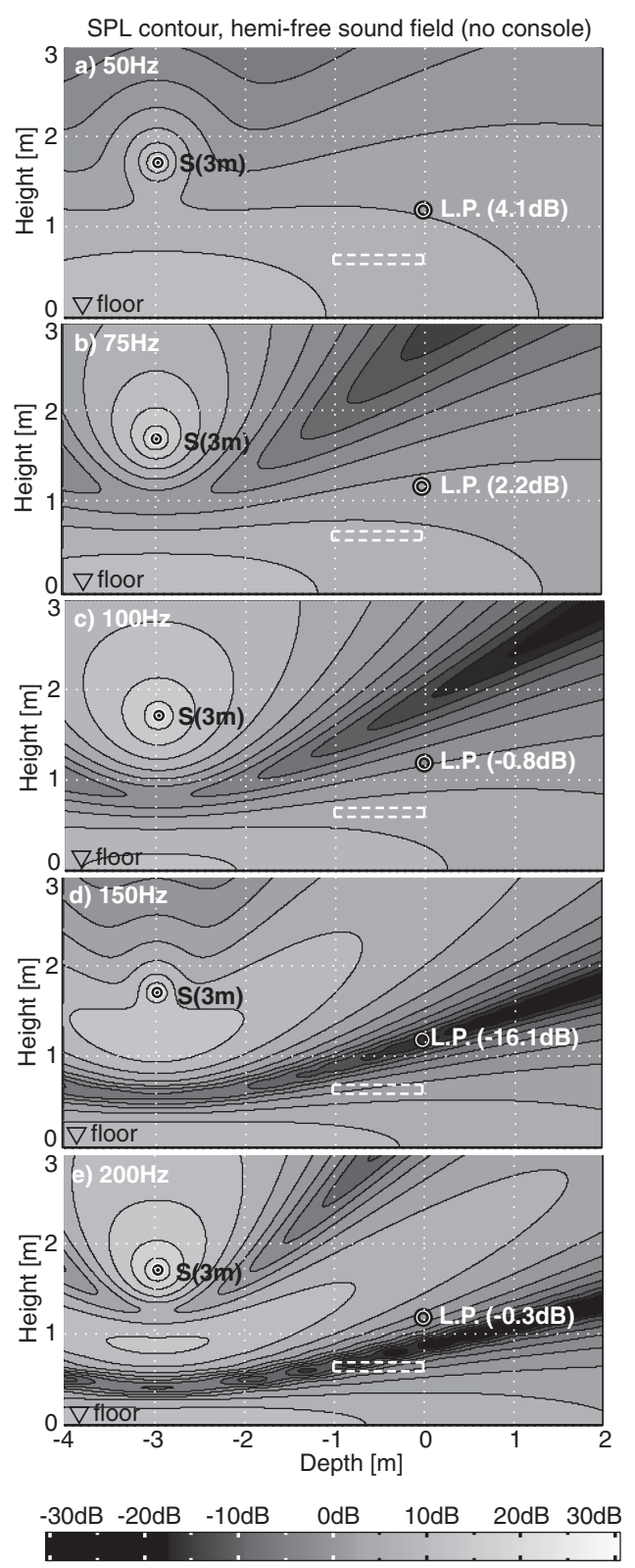

Fig. 16 SPL contours without a mixing console. Results are calculated for a) $50 \mathrm{~Hz}$, b) $75 \mathrm{~Hz}$, c) $100 \mathrm{~Hz}$, d) $150 \mathrm{~Hz}$ and e) $200 \mathrm{~Hz}$ at $0.1 \mathrm{~m} \times 0.1 \mathrm{~m}$ mesh points. The $0 \mathrm{~dB}$ indicates the SPL in a free sound field. Contour lines are plotted at $3 \mathrm{~dB}$ intervals.

segment adheres on the surface of the mixing console; such that one nodal line appears to be generated from the surface of the mixing console.

(3) As the frequency becomes higher, the nodal line which touches with the mixing console appears to revolve around the surface of the mixing console.

(4) The L.P. is involved with the nodal line at approximately $100 \mathrm{~Hz}$. This state is reflected as the dip in the frequency response (Fig. 12, $\mathrm{S}(3 \mathrm{~m})$ ).

In summation it can be said that the variation of the nodal line makes a difference in the dip's appearance between the responses with the mixing console and those without it.

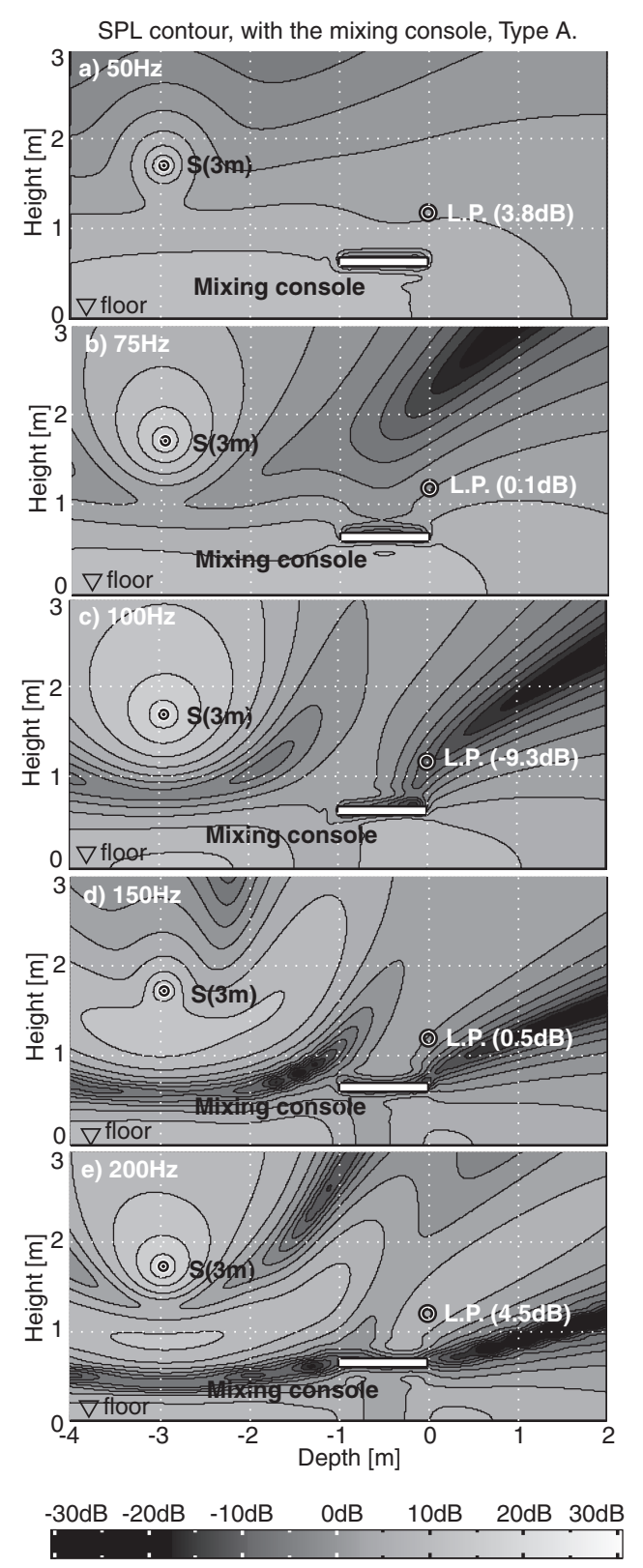

Fig. 17 SPL contours with a Type A mixing console. Results are calculated for a) $50 \mathrm{~Hz}$, b) $75 \mathrm{~Hz}$, c) $100 \mathrm{~Hz}$, d) $150 \mathrm{~Hz}$ and e) $200 \mathrm{~Hz}$ via $\mathrm{BEM}$ at $0.1 \mathrm{~m} \times 0.1 \mathrm{~m}$ mesh points.

\subsection{Contributions from the Real Source and the Imaginary Source}

To examine the contribution from an imaginary source, responses from an imaginary and from a real source were calculated. Figure 18 shows the modeled sound field, where the imaginary source $\left(S^{\prime}(3 m)\right)$ and the imaginary mixing console are inserted instead of the floor.

Figures 19 and 20 show the calculated results; the black solid lines indicate the responses from the real source $(S(3 m))$ and from the imaginary source $\left(S^{\prime}(3 \mathrm{~m})\right)$ respectively. The total responses from all sources $\left(\mathrm{S}(3 \mathrm{~m})+\mathrm{S}^{\prime}(3 \mathrm{~m})\right)$ are also shown as the gray solid lines in Figs. 19 and 20; where solid lines show responses with 


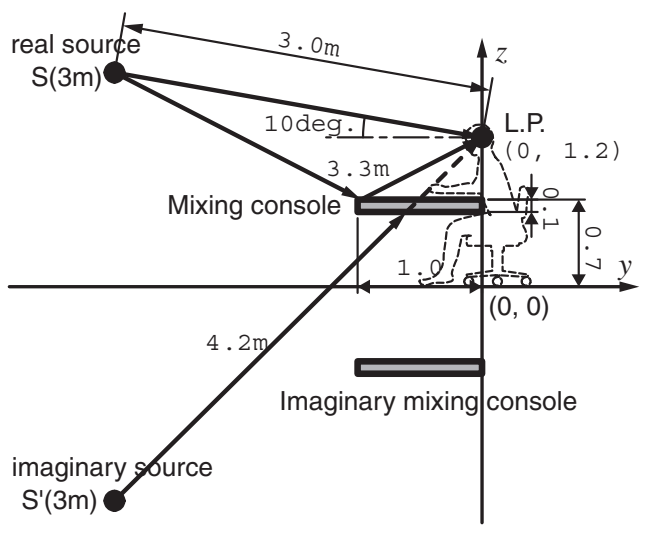

Fig. 18 Modeled sound field without a floor. The imaginary source $S^{\prime}(3 \mathrm{~m})$ and the imaginary mixing console are added instead of the floor.

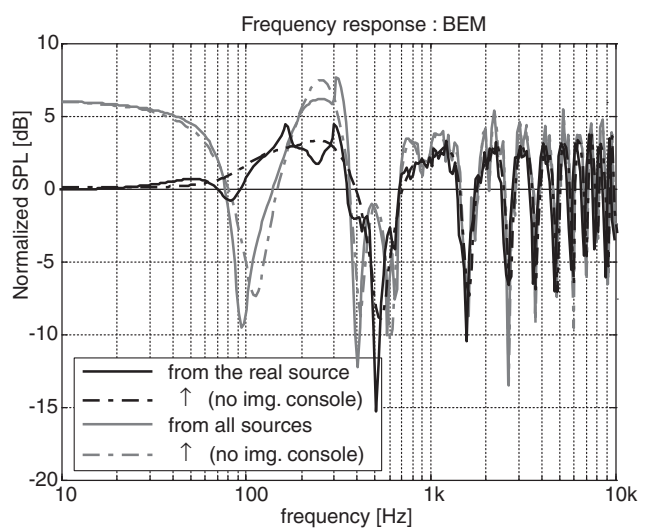

Fig. 19 Calculated responses via BEM. The black lines show the responses from the real source (Fig. 18, $\mathrm{S}(3 \mathrm{~m})$ ), and the gray lines show the total responses from all sources (Fig. 18, $S(3 \mathrm{~m})+\mathrm{S}^{\prime}(3 \mathrm{~m})$ ). The solid lines show the responses with an imaginary mixing console, and the dash-dotted lines show the responses without it.

the imaginary mixing console, and the dash-dotted lines show responses without it.

Comparing the black lines with the gray lines shown in Figs. 19 and 20, the response from the real source and those from the imaginary source (black lines) do not have a dip at around $100 \mathrm{~Hz}$, as is shown in the total response (gray lines). This result suggests that the dip at around $100 \mathrm{~Hz}$ is not just caused by the scattering around the mixing console.

In the response from the real source (Fig. 19, black solid line), periodic dips caused by the path difference between the direct sound $(3.0 \mathrm{~m})$ and the sound reflected from the surface of the mixing console $(3.3 \mathrm{~m})$ are observed. The total level reduces gradually to $0 \mathrm{~dB}$ as the frequency goes lower. This result suggests that the sound reflected from the surface of the mixing console reduces to

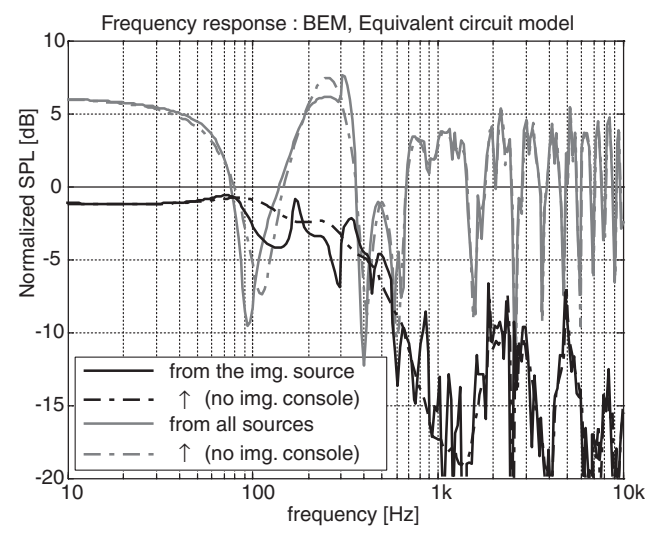

Fig. 20 Calculated responses via BEM. The black lines show the responses from an imaginary source (Fig. 18, $\mathrm{S}(3 \mathrm{~m})$ ), and the gray lines show the total responses from all sources (Fig. 18, $\mathrm{S}(3 \mathrm{~m})+\mathrm{S}^{\prime}(3 \mathrm{~m})$ ). The solid lines show the responses with the imaginary mixing console, and the dash-dotted lines show the responses without it.

be zero at a low frequency, because the finite surface of the mixing console can not yield a complete mirror reflection to the incident sound at low frequencies. In other words, the reflection sound from the surface of a mixing console can be expressed with a high pass filter (HPF), the characteristics of which are determined by the dimensions of the mixing console and the position of the source.

With the response from the imaginary source (Fig. 20, black solid line), the characteristics are almost like a low pass filter (LPF). This result suggests that only low frequency waves can reach the L.P. from the imaginary source.

In respect to the effect of an imaginary mixing console, the responses without the imaginary mixing console (Figs. 19 and 20; dash-dotted lines) show almost the same characteristics as the results with it (solid lines), though a slightly difference is found at the dip of the lowest frequency of the total response (gray lines). Therefore we omitted the effect of the imaginary mixing console in order to simplify the problem.

Figures 21 and 22 show the comparisons of the responses of Types A, B, and S mixing consoles. Figure 21 shows the responses from the real source (Fig. 18, $\mathrm{S}(3 \mathrm{~m})$ ), and Fig. 22 shows the responses from the imaginary source (Fig. 18, $\mathrm{S}^{\prime}(3 \mathrm{~m})$ ). Since the little difference is observed between the responses with the imaginary mixing console and those without it, only the results without the imaginary mixing console are shown. The black solid lines, the gray solid lines and the black dashed lines indicate the responses of Types A, B, and S, respectively. According to the results shown in Figs. 21 and 22, the difference of the shapes among Types A, B, and S, has little effect on both responses from the real source and from the imaginary source, as well as the total response (Fig. 9). Therefore, the 


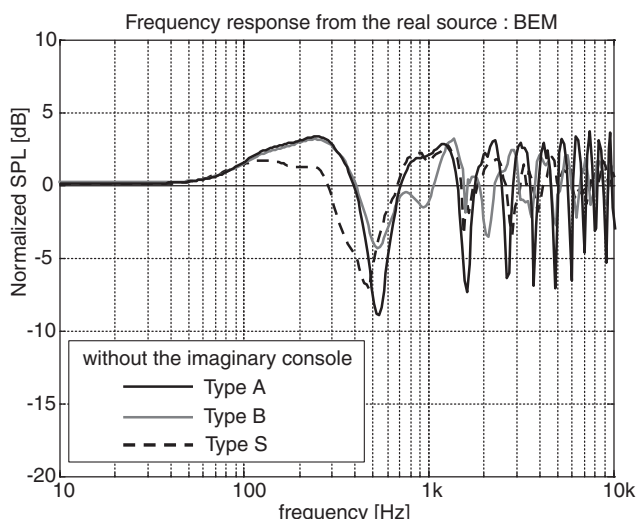

Fig. 21 Calculated responses from the real source (Fig. 18, S(3 m)). The imaginary mixing console is omitted. The black solid line, the gray solid line, the black dashed line show the responses of the 'Type A,' 'Type B,' 'Type S' mixing console, respectively.

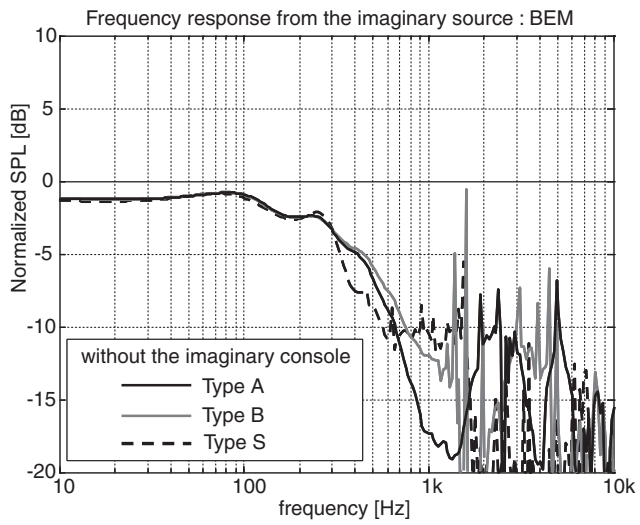

Fig. 22 Calculated responses from the imaginary source (Fig. 18, $\mathrm{S}^{\prime}(3 \mathrm{~m})$ ). The imaginary mixing console is omitted.

responses of Types $\mathrm{A}, \mathrm{B}$, and $\mathrm{S}$ are considered to be generated by the same mechanism.

In conclusion, it can be thought that the mixing consoles behave like a HPF for a field higher than it and like a LPF for a field lower than it.

\subsection{Equivalent Circuit Model}

In accordance with the considerations in section 4.2 above, the sound filed shown in Fig. 18 is assumed to be simplified to Fig. 23 with an equivalent circuit shown in Fig. 24. The imaginary mixing console is omitted in this model. In Fig. 23, S indicates the real source, and $S^{\prime} 1$ and $S^{\prime} 2$ indicate the imaginary sources against the surface of a mixing console and the floor respectively.

In Fig. 24, the transfer functions from $S^{\prime} 1$ or $S^{\prime} 2$ to the L.P. are expressed by three elements, the attenuator, the delay and the filter (HPF, LPF). In the two-dimensional sound field, the direct sound is basically expressed as the

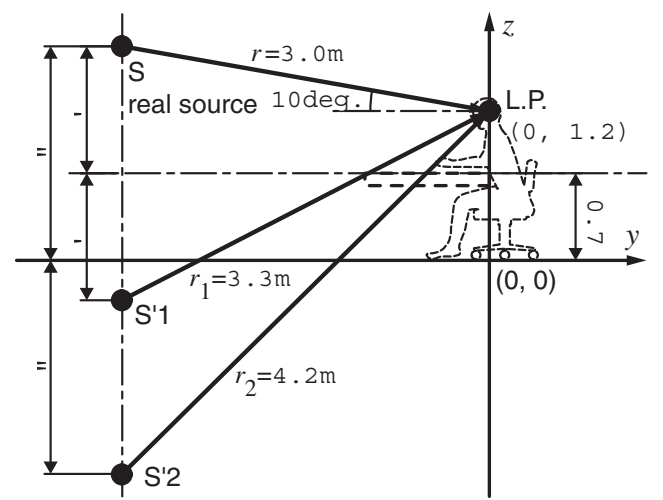

Fig. 23 The simplified field without a mixing console. The secondary imaginary source $S^{\prime} 1$ is added instead of the mixing console.

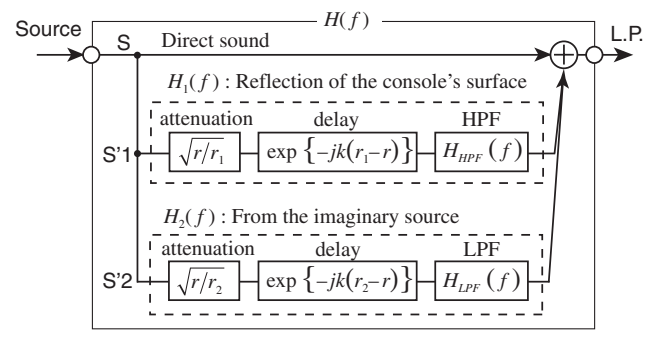

Fig. 24 The equivalent circuit of the sound field shown in Fig. 23.

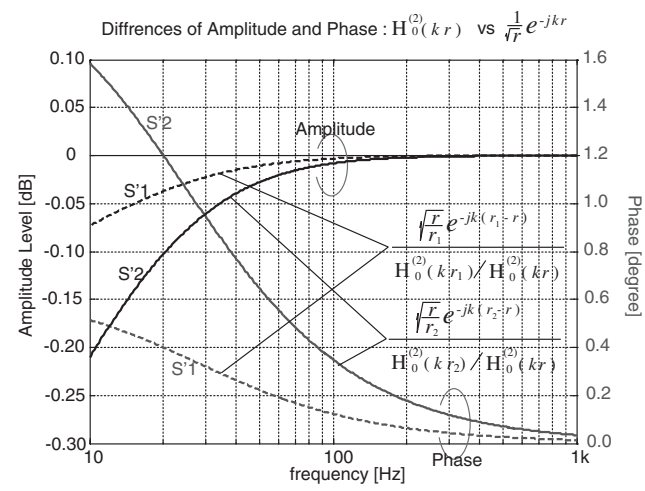

Fig. 25 Differences of the characteristics between the Hankel function of the second kind of order zero, and the exponential function. Black lines show the differences of the amplitude characteristics, and gray lines show the differences of the phase characteristics. The dashed lines and the solid lines show the values when the source is located at $\mathrm{S}^{\prime} 1$ and $\mathrm{S}^{\prime} 2$ (Fig. 23), respectively. All results are normalized by the direct sound (Fig. 23, S).

Hankel function of the second kind of order zero. This expression can be approximated by the exponential phase shift and the attenuation inversely proportional to the square root of the distance from the source.

Figure 25 shows the degree of the approximation, i.e., 
the differences of the amplitude and phase characteristics between the Hankel function and the exponential expression under the conditions assumed here. As the result, the absolute differences of the amplitude and the phase are less than approximately $0.2 \mathrm{~dB}$ and 1.6 degrees at higher than $10 \mathrm{~Hz}$, respectively, and they are considered to be little enough to be ignored. Therefore, the author expresses the source of the circuit model (Fig. 24, $S^{\prime} 1, S^{\prime} 2$ ) in the exponential function instead of the Hankel function for the convenience of the calculation.

The transfer function of the equivalent circuit $H(f)$ is described as follows.

$$
H(f)=1+H_{1}(f)+H_{2}(f)
$$

The unity in the right hand side represent the transfer function of the direct sound; $H_{1}(f)$ and $H_{2}(f)$ represent the transfer functions of the reflection sounds from the surface of the mixing console and from the floor respectively. $H_{1}(f)$ and $H_{2}(f)$ are described in a frequency domain as follows.

$$
\begin{aligned}
& H_{1}(f)=\sqrt{r / r_{1}} e^{-j k\left(r_{1}-r\right)} \cdot H_{\mathrm{HPF}}(f) \\
& H_{2}(f)=\sqrt{r / r_{2}} e^{-j k\left(r_{2}-r\right)} \cdot H_{\mathrm{LPF}}(f)
\end{aligned}
$$

$r, r_{1}$ and $r_{2}$ indicate the distances from $\mathrm{S}, \mathrm{S}^{\prime} 1$ and $\mathrm{S}^{\prime} 2$ to the L.P., respectively. $H_{\mathrm{HPF}}(f)$ and $H_{\mathrm{LPF}}(f)$ are the transfer functions of the second order High Pass Filter and the Low Pass Filter, respectively. They are described by the resonance frequency $f_{0}$ and the quality factor $Q$.

$$
\begin{aligned}
& H_{\mathrm{HPF}}(f)=\frac{1}{1-\left(\frac{f_{0}}{f}\right)^{2}-j \frac{1}{Q}\left(\frac{f_{0}}{f}\right)} \\
& H_{\mathrm{LPF}}(f)=\frac{1}{1-\left(\frac{f}{f_{0}}\right)^{2}+j \frac{1}{Q}\left(\frac{f}{f_{0}}\right)}
\end{aligned}
$$

where, $f_{0}$ and $Q$ are estimated by a series of calculations of the responses from the real and imaginary sources to fit the results via an equivalent circuit to that via the BEM. As a result, $\left(f_{0}, Q\right)=(30 \mathrm{~Hz}, 0.1)$ was assumed for $\mathrm{HPF}$ and $\left(f_{0}, Q\right)=(500 \mathrm{~Hz}, 0.5)$ for LPF. The calculated results via the equivalent circuit using these $f_{0} \mathrm{~s}$ and $Q \mathrm{~s}$ are shown as the black solid lines in Figs. 26 and 27; where the gray lines indicate the calculated results without the imaginary mixing console via the BEM. Also in Fig. 26, the amplitude characteristics of the HPF are shown as the black dashed line. From Figs. 26 and 27, the responses via the equivalent circuit (black solid lines) can be seen to be in good agreement with the responses via the BEM (gray solid lines).

The amplitude characteristics of the HPF and LPF that are used for the equivalent circuit are shown in Fig. 28; where the HPF and LPF seem to compose the cross over filter. This shows that the total energy of all the reflection

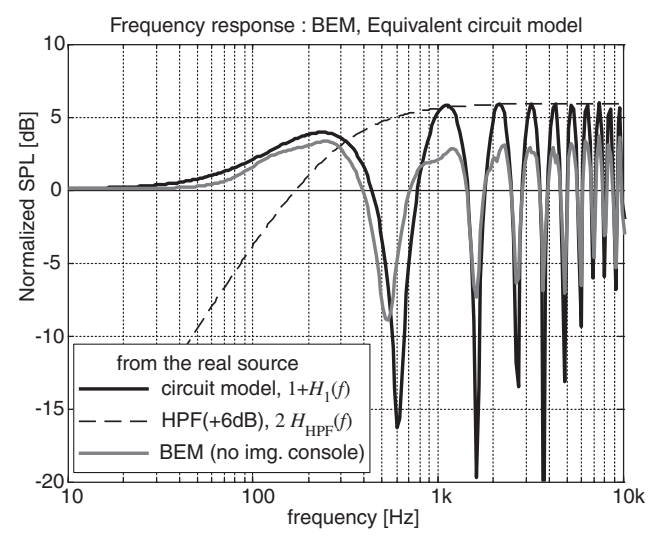

Fig. 26 Calculated responses from a real source (Fig. 18; $\mathrm{S}(3 \mathrm{~m})$, Fig. 23; $\left.\mathrm{S}+\mathrm{S}^{\prime} 1\right)$. The imaginary mixing console is omitted. The black solid line shows the response calculated by the equivalent circuit, and the gray solid line shows the response via BEM. The black dashed line shows the amplitude characteristics of the HPF plotted with $+6 \mathrm{~dB}$ gains.

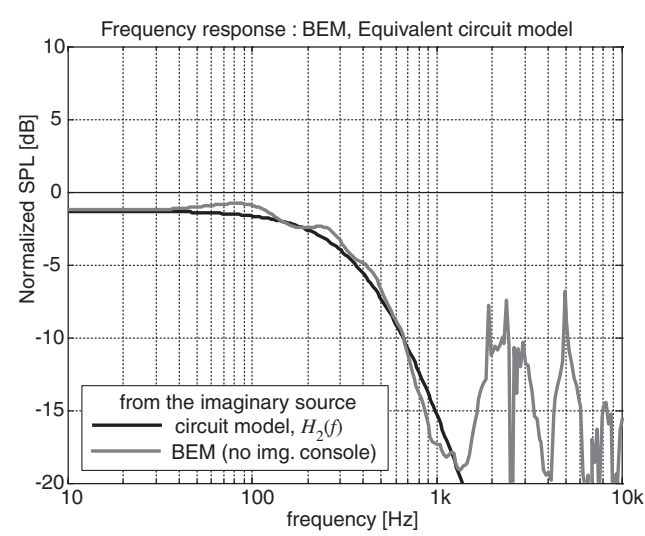

Fig. 27 Calculated responses from an imaginary source (Fig. 18; $\mathrm{S}^{\prime}(3 \mathrm{~m})$, Fig. 23; $\mathrm{S}^{\prime} 2$ ) with no imaginary mixing console. The black solid line shows the response calculated by the equivalent circuit, and the gray solid line shows the response via BEM.

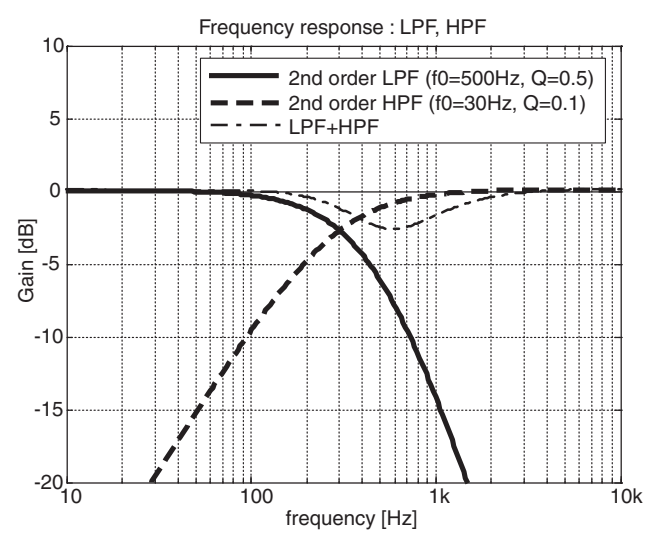

Fig. 28 Amplitude characteristics of the HPF and the LPF that are used in the circuit shown in Fig. 24. 


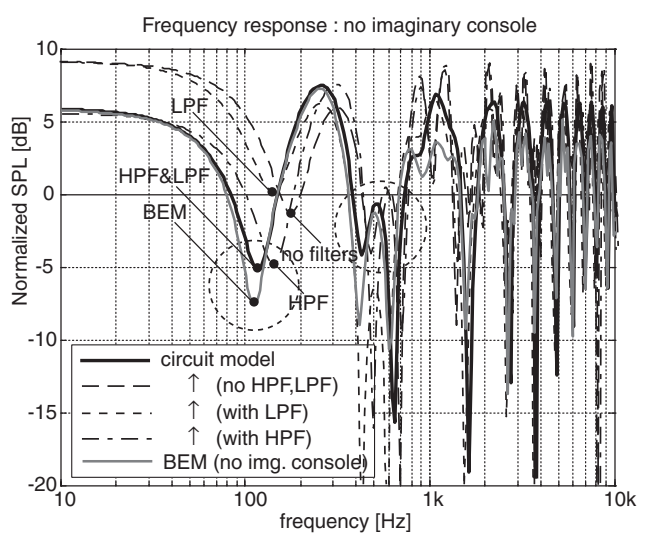

Fig. 29 Final responses calculated by two different methods, BEM (gray line) and the equivalent circuit model (black lines). Contributions from all sources (Fig. 18; $\mathrm{S}(3 \mathrm{~m})+\mathrm{S}^{\prime}(3 \mathrm{~m})$, Fig. $\left.23 ; \mathrm{S}+\mathrm{S}^{\prime} 1+\mathrm{S}^{\prime} 2\right)$ are summed. There is no imaginary mixing console.

sounds is almost same as that of the one reflection sound at the L.P.

The final response, namely the total response that includes the contributions from all sources (Fig. 18; $\mathrm{S}(3 \mathrm{~m})+\mathrm{S}^{\prime}(3 \mathrm{~m})$, Fig. $\left.23 ; \quad \mathrm{S}+\mathrm{S}^{\prime} 1+\mathrm{S}^{\prime} 2\right)$, is shown in Fig. 29. The black solid line indicates the result via the equivalent circuit, while the gray solid line indicates the result without the imaginary mixing console via the BEM. Comparing the two results, it can be seen that they correspond enough well, especially at the low frequency to confirm the validity of the equivalent circuit. Consequently, we can ascertain that the mixing console in the hemifree sound field is expressed by the simple equivalent circuit shown in Fig. 24, and also that the equivalent circuit shows the outline of the generating mechanism of the dips at the low frequency.

To consider the effect of the HPF and the LPF, responses with or without the HPF and/or the LPF was also calculated via the equivalent circuit. Fig. 29 shows the results, the black dashed line presents the response without both the HPF and the LPF; the black broken line presents the response only with the LPF; and the black dash-dotted line represents the response only with the HPF.

Comparing them with the target response calculated via the BEM (gray solid line), they cannot express the appearance of the target response well especially at low frequency. This result suggests that both the HPF and the LPF are the important elements to simulate the low frequency response, and the generating of the dip at low frequency is affected not only by the low passed sound from the floor but also by the high passed sound from the surface of the mixing console. In terms of the little amplitude of the HPF at low frequency, it can be considered that the phase characteristics of the HPF give a larger effect to the dip at around $100 \mathrm{~Hz}$ than its amplitude characteristics.

In conclusion, through examinations via the simple equivalent circuit model, we ascertained that:

(1) The interference of three kinds of sound, 1) direct sound from the source; 2) reflected high frequency sound from the surface of a mixing console; and 3) reflected low frequency sound from the floor, generate the dip in the monitoring response at around $100 \mathrm{~Hz}$.

(2) The behavior of the mixing console as a HPF and a LPF causes the variation in the dip's appearances at low frequency.

Since Types A, B and S mixing consoles show the same kind of the responses (Figs. 12, 21 and 22), the circuit model (Fig. 24) may have a possibility to be applied for many shapes of the mixing consoles. However, in order to clear this point, more detail analyses must be conducted. The authors are continuing to research the relationship between the parameters of the sound filed including the shape of the mixing console and the values of $f_{0}$ and $Q$ under various conditions.

\subsection{Basic Trials in Order to Improve the Low Frequency Response}

We have ascertained, as presented above, that the characteristics of the dip at around $100 \mathrm{~Hz}$ are closely related to the sound reflected from the floor and the presence of the mixing console. Therefore, reduction of these effects should lead the reduction of the dip. Some examples for improving the dip are tried via the BEM in this section. Improvements were carried out by variations of part of the boundary admittances.

When we consider methods to improve, the following must be considered:

- It is almost impossible to physically absorb the top surface of the mixing console.

- Taking account of architectural matters, it is also almost impossible to absorb the whole floor with much sound absorption.

Considering the above, the following two methods were examined (Fig. 30).

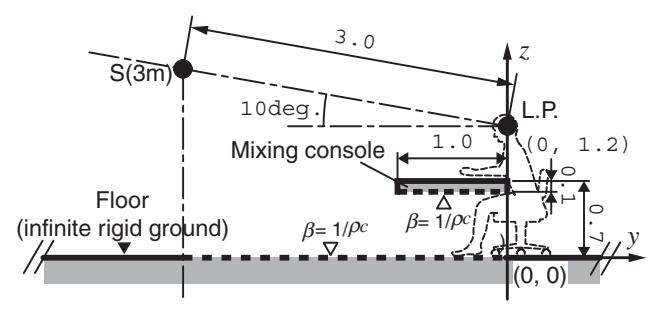

Fig. 30 An experimental model to improve low frequency response. Part of the floor or the bottom of the mixing console is absorbed. Calculated results are shown in Figs. 31 and 32. 
(1) Absorbing the front part of the floor in order to reduce the effect of the sound reflected from the floor. The admittance of $1 / \rho c$ is given at the floor between the L.P. and the source.

(2) Absorbing the bottom of the mixing console to reduce the effect of the presence of the mixing console. The admittance of $1 / \rho c$ is given at the bottom of the console where the reflected sound from the floor incidents.

Figures 31 and 32 show the calculated results of frequency responses and SPL contours at $100 \mathrm{~Hz}$, respectively.

In respect to absorbing part of the floor, this much increases the SPL of the dip at around $100 \mathrm{~Hz}$ (Fig. 31,

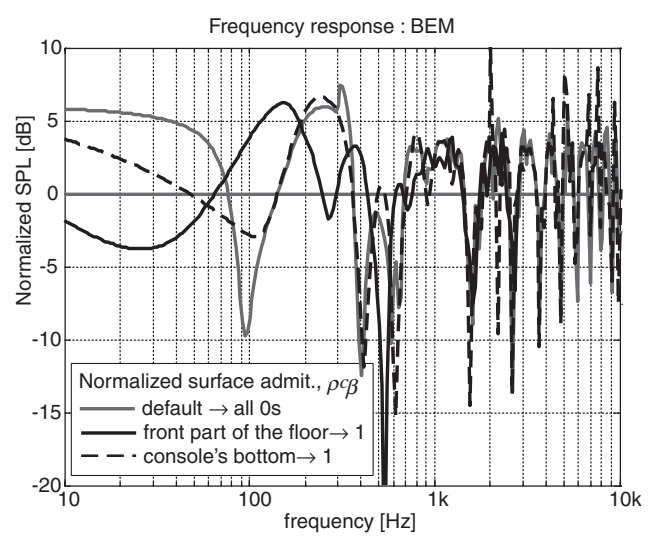

Fig. 31 Frequency responses at the L.P.
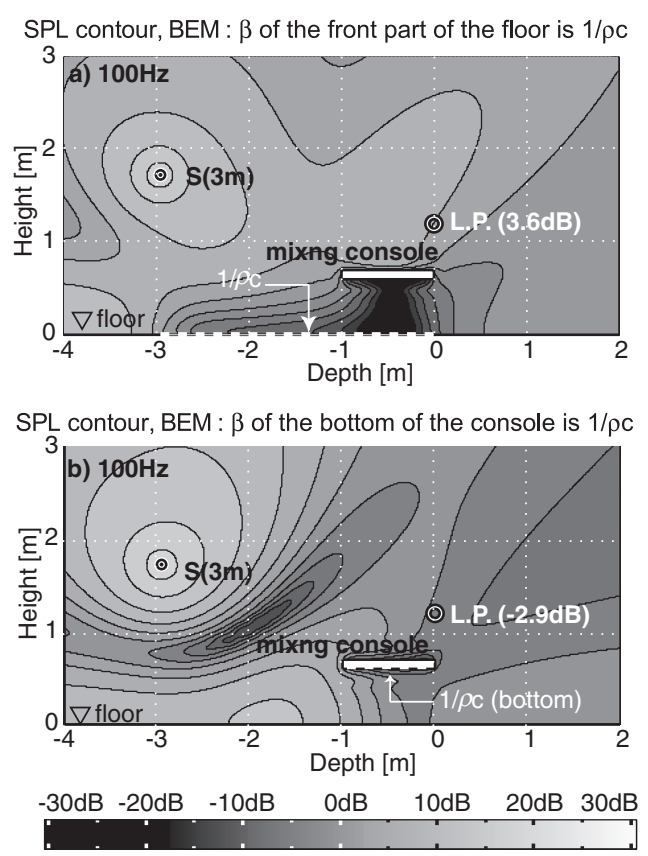

Fig. 32 SPL contours of $100 \mathrm{~Hz}$ calculated via BEM at $0.1 \mathrm{~m} \times 0.1 \mathrm{~m}$ mesh points. a) shows the result with the partial absorbing of the floor; b) shows the result with the absorbing of the console's bottom. black solid line). However the response is no longer the original one (Fig. 31, gray solid line), and it is also different from the response without the imaginary source (Fig. 19, the black solid line). This suggests that absorbing part of the floor cannot sufficiently remove the effect of the imaginary source, and may create another type of response. Consequently this method causes a risk of generating other dips and peaks, such as those at approximately $25 \mathrm{~Hz}$, $150 \mathrm{~Hz}, 250 \mathrm{~Hz}$ and $550 \mathrm{~Hz}$ (Fig. 31, black solid line).

On the other hand, absorbing the console's bottom just affects the low frequency response at less than approximately $110 \mathrm{~Hz}$ (Fig. 31, black dashed line). Though the recovered value of the dip may not be sufficient, an advantage is that new dips and peaks do not appear.

The SPL contours shown in Fig. 32 reflect the appearances of the frequency responses. The SPL distribution with the absorbing of the console's bottom (Fig. 32b) remain the feature of the default field (Fig. 17-c). On the other hand, the SPL distribution with absorbing part of the floor (Fig. 32-a) is no longer the default one (Fig. 17-c), though the nodal line is almost removed.

In conclusion, each method has advantages and drawbacks; a perfect solution is probably hard to find so long as the effect of the imaginary source remains. Detail examinations into combinations of some different kinds of methods are still needed.

\section{CONCLUDING REMARKS}

Dips in the monitoring responses with a mixing console are investigated by three approaches; BEM, 1/10 scaled measurements and the equivalent circuit. The results demonstrated the following (Fig. 33).

(1) The dip affected by the mixing console necessarily occurs at around $100 \mathrm{~Hz}$ in the small/medium sized mixing rooms whose monitoring distances are around $3 \mathrm{~m}$, even though all walls and the ceiling are

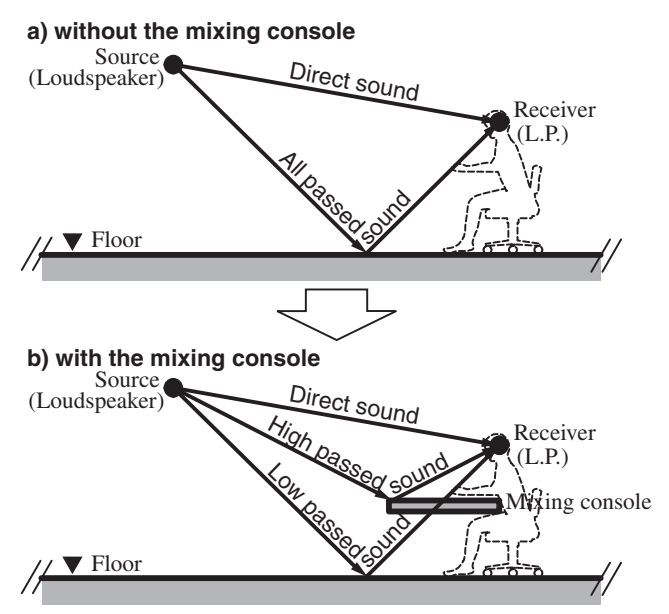

Fig. 33 Images illustrating the effect of a mixing console. 
absorbed perfectly.

(2) Dips are generated by interferences of three kinds of sounds, 1) direct sound from a source; 2) reflected high frequency sound from the surface of a mixing console; 3) reflected low frequency sound from the floor.

(3) The mixing console behaves like a HPF for the sounds that incident from higher than it.

(4) The mixing console behaves like a LPF for sounds that incident from lower than it.

(5) The behavior of the mixing console as a HPF and a LPF affects the characteristics of the dip at low frequency.

(6) Reductions of the reflections on the floor and on the bottom of the mixing console are effective for increasing the SPL at the dip's frequency of around $100 \mathrm{~Hz}$. However, an extreme condition, i.e., perfect absorption, is assumed to reduce the reflected sound; practical examinations are still necessary.

The authors are continuing to research correspondences between the two-dimensional model and the three-dimensional field, and will also investigate the relationship between the effect of the mixing console and the conditions of the room boundary.

\section{ACKNOWLEDGEMENTS}

The author would like to thank Dr. Takashi Ishizuka and Mr. Hisaharu Suzuki of the Kyushu Institute of Design for their comments about the numerical calculations and for the cooperation in the measurements. This study was supported in part by Grant-in-Aid for the 21st Century COE Program.

\section{REFERENCES}

[1] M. Nakahara and A. Omoto, "Room acoustic design for small multichannel studios," Proc. AES 24th Int. Conf. Multichannel Audio, Banff, pp. 319-334 (2003).
[2] M. Nakahara, A. Omoto and K. Fujiwara, "Scattering effect of a mixing console in a production studio," Proc. Int. Symp. Room Acoustics: Design and Science (RADS), 050 (2004).

[3] P. H. Heringa and K. Rijk, "Design of small control rooms with broadband frequency response," Proc. AES 84th Convention, Paris, 2631(K-5) (1988).

[4] P. R. Newell, Project Studios: A More Professional Approach (Focal Press, Oxford, 2000), e.g., pp. 61-84, 146-195.

[5] C. Kai, A. Ikeda, S. Ueoka, M. Nakahara and A. Omoto, "Acoustic design of a multichannel production Studio: Example of a small/medium post-production studio in Tokyo," Proc. Int. Symp. Room Acoustics: Design and Science (RADS), 055 (2004).

[6] Method for the subjective assessment of small impairments in audio systems including multichannel sound systems, Rec. ITUR BS. 1116-1, 16-18 (1994-1997).

[7] K. Mimoto, T. Wakatsuki and M. Sawaguchi, "Multi-channel sound control room in NHK: Design policy and guideline," Proc. AES 19th Int. Conf. Surround Sound, Schloss Elmau, pp. 105-110 (2003).

Masataka Nakahara received the M. Design degree from the Kyushu Institute of Design in 1995, and joined the SONA Corporation thereafter. $\mathrm{He}$ is engaged in the acoustic design of professional studios. He is a member of ASJ, EIC, JAS and AES.

Akira Omoto graduated from the Kyushu Institute of Design, Fukuoka, Japan, in 1987, and received the Ph.D. degree from the University of Tokyo, Japan, in 1995. From 1987 to 1991, he worked as a Research and Development Engineer at Nittobo Acoustic Engineering Co., Ltd., Tokyo. In 1991, he was appointed Research Associate at the Department of Acoustic Design, Kyushu Institute of Design, and was made Associate Professor in 1997. His current research interests include active control and the sound field design in small enclosure.

Kyoji Fujiwara graduated from Kobe University in 1969, and received M. E. degree from Kobe University in 1971. He received Dr. Eng. degree from the University of Tokyo in 1979. He began to work as a Research Associate at the Department of Acoustic Design, Kyushu Institute of Design from 1971, and was promoted to Associate Professor in 1985. Since 1989, he is Full Professor. He is now a member of Acoust. Soc. Jpn., Acoust Soc. Am, Architectural Inst. of Jpn., and INCE/Japan. 